\title{
Evaluación de una experiencia de capacitación para el desarrollo de recursos educativos digitales a docentes universitarios
}

\author{
Diana Marcela Cardona-Román* \\ Norma Candolfi-Arballo** \\ Jenny Marcela Sánchez-Torres ${ }^{\star * *}$
}

* Investigadora, becaria Colciencias del Doctorado en Ingeniería: Industria y Organizaciones. Facultad de Ingeniería, Universidad Nacional de Colombia, Bogotá, Colombia.

Correo electrónico:

dmcardonar@unal.edu.co

** Estudiante del Doctorado en Sistemas y Ambientes Educativos. Profesora, Centro de Ingeniería y Tecnología, Unidad Valle de las Palmas, Universidad Autónoma de Baja California, Tijuana, México. Correo electrónico: ncandolfi@uabc.edu.mx

*** Doctora en Economía y Gestión de la Innovación y Política Tecnológica, Universidad Autónoma de Madrid. Profesora titular, Departamento de Ingeniería de Sistemas e Industrial, Facultad de Ingeniería, Universidad Nacional de Colombia, Bogotá, Colombia. Correo electrónico:

jmsanchezt@unal.edu.co

Recibido: 12 de diciembre del 2015

Aprobado: 21 de febrero del 2016

Cómo citar este artículo: Cardona-Román, Diana Marcela, Norma Candolfi-Arballo y Jenny Marcela Sánchez-Torres. "Evaluación de una experiencia de capacitación para el desarrollo de recursos educativos digitales a docentes universitarios". Rastros Rostros 18.32 (2016): 11-27. Impreso. doi: http:// dx.doi.org/10.16925/ra.v18i32.1315

\section{Resumen}

Introducción: como una estrategia de colaboración interinstitucional entre México y Colombia, se propuso evaluar los resultados de una capacitación en recursos educativos a docentes del Centro de Ingeniería y Tecnología de la Universidad Autónoma de Baja California. Metodología: el tipo de investigación empleado fue descriptivo con un enfoque mixto. Los datos se recolectaron por encuesta con un instrumento de 13 preguntas y una muestra intencional de 10 docentes. Resultados: se definieron ocho variables (género, edad, modalidad de enseñanza, expectativas, utilidad, motivación, aprendizaje de los estudiantes y herramientas), se evaluó la consistencia interna del instrumento (coeficiente de Pearson de 0,879, alfa de Cronbach de 0,947) y se mostró que una formación planificada con necesidades reales impacta positivamente a los docentes. Conclusiones: la planificación y asistencia a capacitaciones aumenta la motivación y el interés de los docentes. También propicia un ambiente favorable para la incorporación y el uso de recursos didácticos en las aulas virtuales como estrategia de enseñanza.

Palabras clave: capacitación, evaluación de la tecnología, formación de docentes, recursos educacionales, universidad. 


\title{
Evaluation of a training experience for university professors in the development of digital educational resources
}

\begin{abstract}
Introduction: The evaluation of the results of a training in educational resources for teachers from the Engineering and Technology Center at the Universidad Autónoma de Baja California was proposed as a strategy of inter-institutional collaboration between Mexico and Colombia. Methodology: The type of research used was descriptive with a mixed approach. Data were collected using a survey instrument of 13 questions and a purposive sample of 10 teachers. Results: Eight variables (gender, age, teaching method, expectations, relevance, motivation, student learning, and tools) were defined, the internal consistency of the instrument (Pearson correlation coefficient of 0.879 , Cronbach's alpha of 0.947 ) was evaluated, and it was found that a training designed to meet real needs has a positive impact on teachers. Conclusions: The planning of and participation in trainings increases the motivation and interest of teachers. Trainings also foster a favorable environment for the incorporation and use of teaching resources in virtual classrooms as a teaching strategy.
\end{abstract}

Keywords: training, technology assessment, teacher training, educational resources, university.

\section{Avaliação de uma experiência de capacitação para o desenvolvimento de recursos educativos digitais a docentes universitários}

\section{Resumo}

Introdução: como uma estratégia de colaboração interinstitucional entre México e Colômbia, propôs-se avaliar os resultados de uma capacitação em recursos educativos a docentes do Centro de Engenharia e Tecnologia da Universidad Autonóma de Baja Califórnia. Metodologia: o tipo de pesquisa utilizado foi descritivo com um enfoque misto. Os dados foram coletados por meio de uma entrevista com 13 perguntas e uma amostra intencional de 10 docentes. Resultados: foram definidas oito variáveis (gênero, idade, modalidade de ensino, expectativas, utilidade, motivação, aprendizagem dos estudantes e ferramentas), avaliou-se a consistência interna do instrumento (coeficiente de Pearson de 0,879, alfa de Cronbach de 0,947 ) e mostrou-se que uma formação planejada com necessidades reais impacta positivamente os docentes. Conclusões: o planejamento e o comparecimento nas capacitações aumentam a motivação e o interesse dos docentes. Também propicia um ambiente favorável para a incorporação e o uso de recursos didáticos nas aulas virtuais como estratégia de ensino.

Palavras-chave: capacitação, avaliação da tecnologia, formação de docentes, recursos educacionais, universidade. 


\section{Introducción}

El desarrollo de capacidades de capital humano y la generación de innovación son indicadores importantes dentro de las nuevas propuestas de modelos de crecimiento de la economía de un país. Habida cuenta de que esto permite tener individuos con mayores habilidades que potencien el conocimiento adquirido orientándolo a la mejora continua y al desarrollo personal. Así mismo, tener comunidades conscientes de su entorno contribuye a que estas planteen soluciones a las necesidades y problemáticas regionales, soluciones que impacten positivamente en el progreso de una nación.

Los avances tecnológicos han generado la necesidad de ser incluidos en los procesos de enseñanza-aprendizaje, y esto hace que los modelos educativos sobrelleven constantes cambios que trascienden si los dirigentes de las unidades académicas promueven y socializan entre sus docentes la capacitación e incorporación de tecnologías en su labor. Para esto es necesario que se tengan definidas, limitadas y justificadas las actividades a realizar. González planteó que

La era digital ha generado grandes transformaciones y beneficios [...] para alcanzar estos beneficios se requiere acoplar las tecnologías de la información con un replanteamiento claro de los procesos, las estructuras administrativas y la descripción de los trabajos de las personas e instituciones involucradas. (2)

Lo anterior muestra la importancia de incorporar las tecnologías de la información y comunicación (TIC) en un proceso global en el que se consideren todos los actores siguiendo un programa integrador que dé cumplimiento al plan de desarrollo de cada institución. Esto con la finalidad de que cada actor dentro del proceso de incorporación de TIC se involucre y logre acciones de impacto.

En el sector educativo uno de los actores es el docente y para que este integre las TIC dentro de su labor profesional es necesaria una actualización permanente (Vezub 3). En Los desafíos de las TIC para el cambio educativo de la Organización de Estados Iberoamericanos (OEI) se especifica que no basta con dotar tecnológicamente a las instituciones de educación, es necesario también avanzar en la incorporación y uso de las TiC en los entornos educativos para reducir la brecha digital, y para esto hace falta abordar, al mismo tiempo, un cambio en la estructura organizativa de las instituciones educativas, así como en las competencias digitales de los docentes (OEI 154).

En ese sentido, la educación superior es un espacio en el cual las capacitaciones del capital humano se realizan de manera continua, dado que de algún modo las instituciones se transforman y se adaptan a los cambios para hacer frente a las nuevas demandas educativas de la sociedad del conocimiento (González). Para el Banco Interamericano de Desarrollo (BID), la formación docente es un indicador claro de crecimiento institucional. Además, esta entidad clasifica las iniciativas de capacitación en generales y específicas: las primeras son para la adquisición de destrezas generales de uso de las TIC (Severin 14, 27; OEI 145), y las segundas, de entrenamiento y formación, están asociadas al uso específico de las tecnologías con fines y contextos educativos que contribuyen al desarrollo de capacidades tanto personales como institucionales (Severin 14).

La formación docente es una de las estrategias más relevantes para la actualización curricular. Los docentes son el vínculo dentro de las instituciones de educación entre las necesidades sociales, los avances tecnológicos y los contenidos impartidos, de ahí la responsabilidad del docente por actualizarse y capacitarse constantemente (Unesco-IEU; González). Como lo manifiesta Severin (17), aparte de la actualización curricular, es importante que el docente integre las TIC en su labor y adquiera destrezas generales en el uso de tecnologías que le permita aplicarlas en el ámbito educativo.

De acuerdo con lo anterior, los docentes universitarios son agentes de cambio que deberán contribuir en el desarrollo de habilidades laborales, competencias para la vida y capacidades tecnológicas dentro del bagaje de conocimiento de un estudiante, toda vez que ellos son responsables del buen uso y del aprovechamiento de los recursos tecnológicos en el aula de clase (Álvarez et al. 3; Caicedo-Tamayo y Rojas-Ospina 528; González; IDIE; Riascos-Erazo, Quintero-Calvache y Ávila-Fajardo 149; Unesco). Un docente que evoluciona en la aplicación de estrategias innovadoras que incluyan la enseñanza por medio de las TIC tiene la oportunidad de transformar no solo sus prácticas educativas, sino también sus métodos de gestión y administración del conocimiento (González). Así, obtiene información de aplicaciones, técnicas de planeación, evaluación y seguimiento que le permiten con el tiempo, agilizar las actividades administrativas de sus asignaturas (Álvarez et al.). 
Son diversos los papeles que debe asumir un docente dentro del entorno tecnológico. Para Dávila et al., es cambiar de ser instructor a ser mediador (3553). Según Paniagua (3) y IDIE, este tiene que pasar de transmisor de conocimiento a facilitador del aprendizaje. Gisbert, citado por González, indica que los papeles que el docente asume son: a) consultor de información; b) colaborador en grupo; c) trabajador solitario; d) facilitador; e) proveedor de recursos, y f) supervisor académico. Wake, Dysthe y Mjelstad, citados por González, aseguran que el docente es mentor de escritura y un orquestador por las funciones administrativas, pedagógicas, tecnológicas y de enlace que debe realizar. En suma, estas labores pueden ser asumidas por el docente siempre y cuando incorpore la tecnología en sus prácticas pedagógicas $y$ en sus actividades cotidianas.

En el 2008, la Unesco publicó Estándares de competencias en TIC para docentes, texto en el que definió tres enfoques principales dentro de un marco de referencia estándar denominado "marco de plan de estudios", para el desarrollo de competencias en TIC para docentes. Este estaba dirigido específicamente para proveedores de formación profesional de docentes, esto es, para el desarrollo de programas de capacitación docente en instituciones de educación. El marco de referencia especificó como objetivos unos ejes de acción y una matriz de habilidades y de competencias (Unesco 11).

Los tres enfoques principales incluyen:

- Nociones básicas de TIC: capacitación docente para comprender las tecnologías de la información y contribuir tanto al desarrollo económico como al social.

- Profundización del conocimiento: aumento en la capacidad de los educandos, de la fuerza laboral para agregar valor a la sociedad y a la economía. Promueve el uso de TIC más sofisticadas mediante cambios en la profundización de la comprensión del conocimiento.

- Generación de conocimiento: crear conocimiento, innovar y participar en sociedades del conocimiento; esto beneficia la participación cívica, la creatividad cultural y la productividad económica.

La figura 1 contiene la matriz de competencias en TIC para docentes, correspondiente a los estándares de la Unesco. En estos se indican las competencias y habilidades que se buscan desarrollar teniendo en cuenta tres enfoques, a saber, las nociones básicas de TIC, profundización del conocimiento y generación de conocimiento.

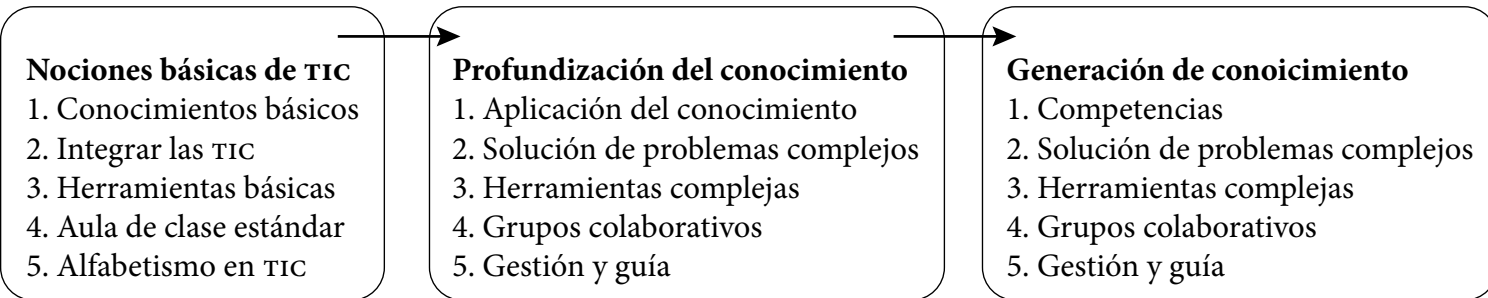

Figura 1. Matriz de competencias de los estándares de la Unesco. Cada una de estas competencias y habilidades se clasifica en cinco componentes según su orden: 1) plan de estudios y evaluación; 2) pedagogía; 3) TIC; 4) organización y administración, y 5) formación profesional de docentes.

Fuente: Unesco. "Aumento de las competencias de los docentes en materia de Tic gracias a los recursos educativos abiertos". Communication \& Information. 3 de julio del 2013: 1. Impreso.

Carneiro, Toscano y Díaz analizan los estándares de la Unesco en oEI (148); en este texto, presentan una versión sintética de estos. Allí se entiende el componente TIC del estándar en el enfoque de nociones básicas como que "[...] los docentes deben conocer el funcionamiento básico del hardware y del software, de aplicaciones de productividad, navegador de Internet, programa de comunicación, presentador multimedia y aplicaciones de gestión". Para el enfoque de profundización del conocimiento, se especifica que "[...] los docentes deben conocer una variedad de aplicaciones y herramientas específicas [o complejas] y deben ser capaces de utilizarlas con flexibilidad en diferentes situaciones basadas en problemas y proyectos [...]" (148).

Como parte del proceso de formación docente del enfoque profundización del conocimiento, se incluyen los recursos educativos digitales (RED), definidos por el 
BID como el material digital destinado a la enseñanza y aprendizaje con uso de medios tecnológicos. Este incluye software educativo, recursos digitales, enciclopedias, manuales, textos escolares, libros, guías, videos, imágenes, hipertextos, etc. (Severin 13). Además de la capacitación a los docentes, es necesario realizar mediciones sobre la incorporación de las TIC en la educación, toda vez que las iniciativas existentes aún son insuficientes. Adicionalmente, hay una carencia de instrumentos específicos que den confianza para medir el impacto tanto de la incorporación de las TiC como de la utilidad de los planes de capacitación docente (Severin).

Es así como en Cardona-Román y SánchezTorres especifican que la evaluación es necesaria en la medida que permite identificar los aspectos débiles y fuertes (en este caso de la formación docente en profundización del conocimiento de TIC), y ayuda a tomar decisiones o acciones de mejora que contribuyan a realizar los ajustes necesarios (RiascosErazo, Quintero-Calvache y Ávila-Fajardo 154-156). Además, el instrumento principal empleado en la evaluación para la recolección de datos es la encuesta (Caicedo-Tamayo y Rojas-Ospina 523; González-Such, Bakieva y García-Bellido; Riascos-Erazo, QuinteroCalvache y Ávila-Fajardo 141).

La experiencia particular que nos compete fue la capacitación para el desarrollo de RED a docentes que orientaban cursos virtuales, la cual fue llevada a cabo en la Escuela de Ciencias de Ingeniería y Tecnología (Ecitec) de la Universidad Autónoma de Baja California (UABC), campus Tijuana, Unidad Valle de las Palmas, en noviembre del 2013. Esta se enmarcó en el segundo enfoque de los estándares de la Unesco, profundización de conocimiento, y en el componente TIC.

La evaluación de la experiencia de capacitación se realizó entre octubre y noviembre del 2014, entre 11 y 12 meses después de efectuada la capacitación para el desarrollo de RED. Esta tuvo el fin de conocer la valoración de los docentes sobre la importancia de las Tic en la educación y el valor de capacitaciones docentes en herramientas tecnológicas que apoyen el proceso pedagógico en la enseñanza universitaria.

El objetivo de este documento es presentar la evaluación sobre la experiencia acerca de la formación docente. Es así que, para exponer los resultados de la evaluación, es necesario describir la experiencia de la capacitación. Por esto esta investigación tuvo dos momentos. El primero de estos fue la capacitación docente impartida en el Taller Básico para crear Recursos Educativos Digitales, en la cual se trató acerca de herramientas informáticas para el enriquecimiento de los cursos virtuales, de manera que se incrementaran los conocimientos del capital humano en una organización educativa y este incluyera las nuevas herramientas en sus aulas de clase. El segundo momento fue la evaluación de la capacitación que buscaba conocer los efectos en la formación docente. El análisis se hace a partir del conocimiento de los docentes, pues estos conforman el capital humano de una organización educativa y son un factor determinante en su acción pedagógica (Dávila et al.).

Este documento se divide en cuatro secciones: 1) "introducción", que contiene los referentes teóricos en los cuales se enmarcó la investigación; 2) "materiales y métodos", que incluye la metodología empleada y la descripción de las tres etapas ejecutadas en la investigación, 3) "resultados", en la cual están los productos de la investigación en cada una de sus tres etapas. En la primera de las secciones hay una descripción de la experiencia de formación docente con la incorporación de las TIC; esta sección incluye tanto la planeación administrativa de la formación como la planeación didáctica, relacionándose con la experiencia de la capacitación. En la segunda sección se exponen las variables empleadas para la evaluación de la experiencia, el diseño del cuestionario y el envío del instrumento a la muestra seleccionada. En la tercera sección, se habla sobre los resultados del análisis de los datos recolectados, la importancia de la formación docente en TIC y los efectos de la formación de los docentes en el uso de las Tic en sus aulas de clase. Por último, se presenta la "discusión y conclusiones", de la investigación.

\section{Materiales y métodos}

El tipo de investigación empleado fue descriptivo con un enfoque mixto, tanto cuantitativo como cualitativo, con la definición de variables y el diseño de un instrumento con preguntas cerradas y abiertas. La investigación descriptiva fue utilizada en ambos enfoques, tanto en la parte cuantitativa a través del análisis estadístico para las preguntas cerradas, como en la parte cualitativa por medio del análisis de texto para las preguntas abiertas (Creswell 218).

Esta investigación tuvo las siguientes etapas:

1. Descripción de la experiencia de formación docente en el uso de las Tic.

2. Definición de variables e instrumentos de medición.

3. Análisis de resultados de la evaluación de la experiencia. 


\section{Descripción de la experiencia de formación docente en el uso y aplicación de las TIC}

En esta etapa se describen la planeación y ejecución de la experiencia en la formación docente por la Ecitec de la UABC. La planeación tuvo dos componentes: uno administrativo y otro didáctico.

La planeación administrativa se enmarcó en el plan de desarrollo institucional de la UABC, que incluyó la capacitación docente y la cual promovió entre los docentes diversos recursos de apoyo y técnicas de incorporación de las TIC diferentes a las herramientas básicas que ya dominaban en su ambiente de enseñanza virtual. Al hacer esto, se contribuyó a que los docentes desarrollaran competencias propias del enfoque de la profundización de conocimiento en el componente Tic según la Unesco.

La planeación didáctica estuvo a cargo de una docente invitada de una institución extranjera (de la Universidad Nacional de Colombia), quien también diseñó e impartió el Taller Básico para crear Recursos Educativos Digitales, con el fin de que los docentes usaran herramientas TIC en ambientes virtuales. Operativamente el enfoque principal fue ampliar el panorama de oportunidades dentro de la Web 2.0 y el software educativo de los docentes; el taller fue impartido el 13 y el 14 de noviembre del 2013.

\section{Definición de variables e instrumento de medición}

En esta se describen las variables y el instrumento desarrollado para evaluar la experiencia de formación docente. Se concibieron las variables a medir como aquellos aspectos característicos del objeto de estudio. Las variables pertinentes para el estudio se relacionan con la caracterización del sujeto de estudio (género, edad y modalidad), así como con las variables motivación, utilidad, aprendizaje de los estudiantes y herramientas.

Posteriormente, se diseñó el cuestionario a partir de las variables identificadas y se determinó la importancia de recoger los datos a través de una encuesta. Luego de esto se consideró población de interés a los docentes a los que se educó. Para esto se utilizó un muestreo no probabilístico intencional (Morone), porque la muestra se compuso de los docentes que participaron del Taller Básico para crear Recursos Educativos Digitales, a quienes se les envió el instrumento por correo electrónico con un recordatorio.
El instrumento estuvo abierto por un periodo de cuatro semanas. La evaluación (envío del instrumento) se ejecutó entre 11 y 12 meses después del taller, entre el 20 de octubre y el 20 de noviembre del 2014.

Se contó con la participación voluntaria de 10 (83\%) docentes de la Ecitec de un total de 12 que asistieron a la capacitación. Aunque la muestra no es significativa, sí es orientativa para señalar las ventajas y desventajas de la capacitación docente en el uso de las herramientas, además de los efectos positivos y negativos, y la utilidad de la formación brindada.

\section{Análisis de resultados de la evaluación de la experiencia}

Una vez recolectados los datos sobre los docentes que asistieron a la capacitación a través de la encuesta diseñada, se procedió, en esta etapa, a analizar los datos recopilados y a describir los resultados encontrados.

Se empleó un análisis estadístico descriptivo, con el cual se realiza una abstracción de la realidad e incluye un análisis reflexivo. El análisis reflexivo implica una meditación por parte del investigador y tiene que ver con una ruptura con el sentido común en la que el investigador debe alejarse por sí mismo de la realidad construida, desviarse de las nociones, del saber inmediato, con el fin de poder llegar a la objetividad (Creswell; Morone) y poder brindar una descripción de la realidad.

\section{Resultados}

\section{Descripción de la experiencia de formación docente en el uso y aplicación de TIC}

Esta etapa desarrolla los elementos que se tuvieron en cuenta tanto para la planeación (administrativa y didáctica) de la formación docente, así como su ejecución.

\section{Planeación administrativa para la formación}

La UABC contiene en su plan de desarrollo institucional del 2011 al 2015 las políticas y estrategias dirigidas a la comunidad universitaria, en las que se destacan los aspectos a atender de forma prioritaria. Dentro de estos, está la formación de los alumnos, que promueve una iniciativa específica según la cual: 
[la] contribución de la UABC [es] la atención a la demanda de educación superior con equidad". Se proponen como acciones puntuales ampliar la oferta educativa con pertinencia social a través del fomento de modalidades de enseñanza semipresencial y a distancia. Asimismo, la política institucional cinco, que busca el mejoramiento de la habilitación del personal universitario, se enfoca en la formación y capacitación del personal académico.

Este propone actualizar a los docentes en habilidades pedagógicas con énfasis en la innovación (UABC).

Con el fin de dar respuesta a las políticas de desarrollo de la UABC en la Ecitec Campus Tijuana, se diseñaron e impartieron actos académicos que favorecieran escenarios de intercambio y actualización docente en TIC en modalidades presenciales, semipresenciales y a distancia a través de la plataforma de Blackboard Learn 9.1. ${ }^{1}$

La capacitación docente en TIC dentro de la Ecitec tiene dos dimensiones:

a. Atención a la primera demanda. Los docentes que pertenecen al grupo de la modalidad virtual, semipresencial y a distancia, quienes tienen un mayor nivel de competencias tecnológicas y una necesidad de actualizarse constantemente para la incorporación de diversas aplicaciones en los cursos que realicen en un corto y mediano plazo.

b. Atención a segunda demanda. Los docentes que imparten clase en modalidad presencial y muestran interés en implementar prácticas innovadoras dentro del aula. En este caso el nivel de competencias tecnológicas va desde uno básico a uno intermedio.

La UABC desarrolló el programa flexible de formación y desarrollo docente (PFFDD), cuyo propósito es la mejora continua de la comunidad docente. En el tema de TIC la oferta se divide en tres momentos (CEA):

1. Cursos asociados al sistema de administración de cursos Blackboard.

a. Blackboard para el trabajo en línea.

b. Taller de herramientas de evaluación en Blackboard.

2. Cursos asociados al diseño de cursos y estrategias apoyadas en Tecnologías de la Información, Comunicación y Colaboración (TICC).

Entorno de aprendizaje virtual oficial de la UABC. a. Diseño instruccional para cursos en línea.

b. Estrategias didácticas apoyadas en TICC.

c. Conducción de cursos en línea.

3. Cursos asociados a las herramientas de libre acceso de Internet para la docencia.

a. Aplicaciones de productividad académica para dispositivos móviles.

b. Classroom: una herramienta de Google para la docencia.

Considerando en ampliar la oferta del PFFDD dentro de la definición de estrategias para la formación, actualización e incursión de las TIC en los procesos de enseñanza-aprendizaje, es importante establecer redes de colaboración y analizar otras instituciones de educación superior. Para esto en la Ecitec se estableció una colaboración para el diseño y desarrollo del Taller Básico para crear Recursos Educativos Digitales con la Universidad de Nacional de Colombia, con el fin de crear redes de colaboración y de satisfacer la quinta política institucional, relacionada con la formación docente.

Para el 2013, la Ecitec tuvo 216 docentes vinculados; de estos, solo 33 orientaban cursos virtuales. Por lo tanto, se concibió que el perfil de los docentes que recibieran la capacitación era pertenecer al grupo de modalidad virtual que impartía cursos semipresenciales y a distancia, es decir, dentro de la atención a primera demanda. La propuesta de ofrecer el Taller Básico para crear Recursos Educativos Digitales buscaba promover en los docentes diversos recursos de apoyo (dentro de la Web 2.0), software educativo y técnicas de incorporación fuera de las herramientas básicas que ya dominaban en su ambiente.

\section{Planeación didáctica para la formación docente}

Cuando se habla de planeación didáctica se tiene en cuenta el trabajo de Alonso Tejada, quien precisa que el diseño del plan de trabajo debe contener los elementos que intervendrán en el proceso de enseñanza-aprendizaje. Estos elementos se encuentran organizados de tal manera que contribuyan y faciliten el desarrollo de habilidades, que modifiquen las actitudes de los aprehendientes, y que se relacionen con el objetivo del curso, con la organización de los contenidos (el eje temático), con las actividades de aprendizaje y con la evaluación de los aprendizajes (Monroy). 
El eje temático sobre el cual giró la capacitación fue la puesta en conocimiento de software para la creación de RED con la intención de desarrollar competencias para la solución de problemas en los docentes y promover un uso de herramientas TIC complejas. La duración planeada de la capacitación fue de 15 horas divididas en cuatro partes.

Una vez definido el eje temático de la capacitación y su duración, se procedió a diseñar su estrategia, la cual tuvo un componente teórico y otro práctico. El componente teórico consistió en una exposición magistral con preguntas a los docentes sobre los conocimientos que tenían del tema. El componente práctico se centró en la explicación de programas informáticos (software), con una sesión permanente de preguntas y respuestas. La explicación empezaba con la descarga de las aplicaciones, la instalación, el uso y el producto. Al terminar la explicación de la aplicación informática se planificó dejar como actividad evaluativa la elaboración de un ejercicio corto con una duración de 15 minutos, de tal manera que fuese un taller dinámico.

Para finalizar la planeación didáctica, se tuvieron en cuenta los recursos necesarios (bibliografía, medios y materiales). Para esto se contó con el uso de Internet, un proyector, equipos de cómputo, diapositivas y aplicaciones informáticas para la creación de los RED. Se notificó acerca de los derechos de autor según correspondió a cada caso, y las diapositivas contaron con diferentes imágenes, videos, referencias bibliográficas e hipervínculos para la ampliación de la información por parte de los docentes.

\section{Desarrollo del taller para la formación docente}

El Taller Básico para crear Recursos Educativos Digitales tuvo dos jornadas. La primera parte de la primera jornada comprendió los aspectos teóricos de los RED, la definición, la clasificación del recurso de acuerdo con el acceso (público, privado, abierto, restringido, pago, gratuito), con la licencia de propiedad intelectual y con el tipo de recurso (información, aprendizaje, colaboración); además de especificar las ventajas de usar RED y las propiedades que generalmente deben tener los objetos de aprendizaje. En la segunda parte de la primera jornada, se definieron los recursos educativos informativos como aquellos que buscan llamar la atención de los estudiantes con contenidos animados y divulgar el conocimiento de manera innovadora, empleando diferentes herramientas digitales para crear anuncios animados, esquemas o representaciones gráficas como mapas conceptuales, mapas mentales o cómics. Estas herramientas pueden ser usadas como recursos educativos colaborativos cuando se indica su uso por parte del docente a un grupo de estudiantes a través de una actividad temática particular.

La segunda jornada abarcó, en la tercera parte, la instrucción y el manejo de herramientas para hacer videos, grabar la pantalla (para el diseño de tutoriales), crear revistas digitales, originar archivos flash, editar imágenes, editar audio. También se buscaba instruir en el uso de herramientas para la creación de recursos educativos de aprendizaje con contenidos procesados y para la elaboración de exámenes con ejercicios de elección múltiple, crucigramas, sopas de letras, emparejamiento, ordenación, completar espacios en blanco, reconstrucción de frases a partir de palabras desordenadas, juegos como el de adivinar una palabra o frase, entre otros. En la cuarta parte, se explicó la forma de incorporar los RED diseñados en una plataforma educativa.

Durante las dos jornadas, se resolvieron las preguntas de los docentes y se diseñaron pequeños ejercicios en los que se aplicaron y subieron algunos de sus recursos en sus cursos virtuales de Blackboard, en especial los informativos. Al final del taller, se les preguntó si la capacitación satisfizo sus expectativas y, de manera unánime, los docentes manifestaron estar de acuerdo y expresaron su alegría de haber participado en ese tipo de actualización.

\section{Definición de variables e instrumento de medición}

\section{Variables de medición}

Para cumplir el objetivo y alcance de esta investigación, se establecieron como variables los aspectos característicos del objeto de estudio. Las variables identificadas como pertinentes para el estudio se mencionan en la tabla 1, la cual incluye además, los ítems del cuestionario y su escala de medición.

Para cada una de las variables, se definió un conjunto de indicadores con escalas de medición nominal y ordinal. Siguiendo a Severin, esto se debe a que el uso de indicadores permite medir el grado de desarrollo y maduración de una estrategia (4); en este caso, este ayudó a medir la utilidad de la capacitación en recursos digitales básicos y su efecto en la labor de enseñanza en aulas virtuales. 
Tabla 1. Clasificación de los ítems del cuestionario aplicado a las variables identificadas del estudio

\begin{tabular}{|l|l|l|}
\hline \multicolumn{1}{|c|}{ Variable } & Ítems & Escala de medición \\
\hline Género & 1 & Dicotómica \\
\hline Edad & 2 & Intervalo \\
\hline Modalidad de enseñanza & 3 & Categórica \\
\hline Expectativas & 4 & Abierta \\
& 5 & Dicotómica \\
\hline Utilidad de la capacitación & 6 & Escala 1-5 \\
\hline Motivación & $10 \mathrm{~g}, 11$ & Escala 1-5 \\
& 12 & Dicotómica \\
\hline Aprendizaje de los estudiantes & 10 & Escala 1-5 \\
\hline $\begin{array}{l}\text { Herramientas (facilidad de uso, }, \\
\text { utilidad, impacto) }\end{array}$ & $7,8,9$ & Escala 1-5 \\
\hline
\end{tabular}

Fuente: elaboración propia.

El propósito de las variables "género", "edad" y "modalidad de enseñanza" fue identificar a los sujetos de estudio. La variable "expectativas" permitió determinar la disposición e intención de recibir el taller que tenían los docentes y si el desarrollo de este cumplió con lo esperado por ellos. Cuando un aprendiz incorpora la nueva información y la transforma en conocimiento, lo hace porque se encuentra motivado, razón por la cual se propuso la variable "motivación”, y además esto aparece en el Segundo estudio sobre información tecnológica en educación 2006 en el que se evaluó el concepto "desarrollo de personal" midiendo la "[...] motivación o exigencias para que los docentes adquirieran el conocimiento y las competencias relacionadas con la práctica pedagógica y la utilización de TIC" (Unesco-IEU 19).

La intención de la variable "utilidad de la capacitación" fue valorar el cumplimiento de las expectativas y la aplicación de lo aprendido en cada curso que el docente orientó en un ambiente en línea. La variable "aprendizaje de los estudiantes" describió el conocimiento de los docentes frente al cambio de actitud de los estudiantes, el interés, las expectativas, el desarrollo de pensamiento crítico, la comunicación, la creatividad y la innovación en la solución. La variable "herramientas" midió la facilidad de uso de los programas informáticos en los cuales los docentes recibieron formación, la utilidad en el curso orientado y el resultado en el aprendizaje de los estudiantes.

\section{Instrumento de medición}

Se utilizó como instrumento de recolección de datos la encuesta publicada en línea, lo que permitió que los docentes diligenciaran el cuestionario desde donde se encontraran. Además eran libres de manifestar su opinión puesto que la encuesta fue anónima y no tenía la presión de la presencia del encuestador.

La encuesta se diseñó con 13 preguntas o ítems con la intención de medir la percepción de utilidad y el efecto de la capacitación en herramientas digitales en los docentes. El instrumento tenía cuatro partes: la primera estuvo relacionada con datos generales del sujeto, es decir, con la caracterización; la segunda tuvo en cuenta las expectativas del docente y la utilidad de la capacitación, esto es, la calidad de la capacitación; la tercera albergó la medición del uso de las herramientas digitales (recursos de apoyo) por parte de los docentes en los cursos que orientó, y la cuarta parte tuvo preguntas acerca del comportamiento académico de los estudiantes desde la perspectiva del docente, así como sobre el comportamiento del docente en el uso de los programas informáticos, con el fin de determinar el efecto de la capacitación.

El instrumento tuvo dos preguntas abiertas opcionales, denominadas "expectativas de la capacitación" y "observaciones generales del tema de la encuesta”; tres preguntas con opción de respuesta dicotómica; una pregunta con una respuesta categórica; una pregunta con una respuesta tipo intervalo, y las seis preguntas restantes tuvieron opciones de respuestas escala tipo Likert de 1 a 5 .

Se diseñó el instrumento en Google Apps (Forms) y se envió a través del correo electrónico a los docentes que participaron de la capacitación de herramientas digitales (también porque les convenía a ellos). En el mensaje se les invitaba a participar de la actividad y se les exponía la intención de la investigación. Además de esto se envió un correo recordatorio para el diligenciamiento de la encuesta.

El instrumento tuvo un índice de correlación de Pearson de 0,8798016227 y el alfa de Cronbach de 0,9477932071, lo que garantizó su consistencia interna y confiabilidad.

\section{Análisis de resultados de la evaluación de la experiencia}

Teniendo en cuenta las respuestas al cuestionario provistas por los docentes de la modalidad virtual de la Ecitec, se comprobó que estas estuvieran completas y que el patrón de respuestas indicara que el encuestado sí entendió las instrucciones de diligenciamiento. Además se revisó la variación de las respuestas, esto es, que el encuestado no haya dado la misma respuesta a todas las preguntas, lo que permitió establecer que 
todas las respuestas recolectadas fueron aceptadas para analizar. En la tabla 2 se muestra la ficha técnica de la encuesta realizada. Posteriormente se realizó un análisis estadístico descriptivo para obtener conclusiones generales respecto del resultado de la capacitación docente en la UABC, y se hizo un análisis reflexivo sobre la utilidad de los planes de formación institucional y de las competencias de los docentes en la enseñanza con apoyo de las Tic.

Tabla 2. Ficha técnica de la evaluación de la experiencia

\begin{tabular}{|l|l|}
\hline \multicolumn{2}{|c|}{ Ficha técnica de la evaluación de la experiencia } \\
\hline Universo & $\begin{array}{l}33 \text { docentes de modalidad virtual de } \\
\text { la Ecitec }\end{array}$ \\
\hline Población & 12 docentes participantes del taller \\
\hline Tamaño de la muestra & 10 docentes participantes del taller \\
\hline Nivel de confianza & $95 \%$ \\
\hline $\begin{array}{l}\text { Procedimiento de } \\
\text { muestreo }\end{array}$ & $\begin{array}{l}\text { muestreo no probabilístico - inten- } \\
\text { cional }\end{array}$ \\
\hline Periodo de realización & Octubre y noviembre del 2014 \\
\hline Tipo de instrumento & $\begin{array}{l}\text { Cuestionario estructurado tipo } \\
\text { encuesta }\end{array}$ \\
\hline
\end{tabular}

Fuente: elaboración propia.

\section{Estadísticos de las preguntas con respuesta tipo escala}

Se respondió la encuesta por 10 docentes de los 12 que participaron en el Taller Básico para crear Recursos Educativos Digitales, dictado en la UABC, es decir, el $83 \%$ de la población. Aunque la muestra no es significativa, sí es orientativa para señalar las ventajas o desventajas del taller, sus efectos positivos o negativos, y su utilidad.

Antes de iniciar el análisis descriptivo de los resultados, se presentan en la tabla 3 los estadísticos básicos de las preguntas valoradas con escala tipo Likert de 1 a 5 , en los que 1 es el valor más bajo o negativo y 5 , el más alto o positivo. Se incluyen las variables identificadas, la mediana de los resultados, el valor mínimo, el valor máximo, la varianza y la desviación estándar; esta última mide el grado de dispersión de las observaciones individuales.

Los resultados obtenidos son de carácter descriptivo y están estructurados en torno a las diferentes variables en la que se ha dividido el cuestionario utilizado (ver tabla 1). Se destacan algunos ítems que consideramos de relevancia para la consecución del objetivo del trabajo de investigación.

Tabla 3. Estadísticos de las preguntas con respuesta tipo escala

\begin{tabular}{|c|c|c|c|c|c|c|}
\hline Variable & Pregunta & Mediana & Varianza & $\begin{array}{c}\text { Desviación } \\
\text { Estándar }\end{array}$ & Mínimo & Máximo \\
\hline Utilidad de la capacitación & 6 & 5 & 0,450 & 0,671 & 3 & 5 \\
\hline Facilidad de uso de las herramientas & 7 & 4 & 0,667 & 0,770 & 1 & 5 \\
\hline Utilidad de las herramientas en el curso que orienta & 8 & 4 & 0,827 & 0,879 & 1 & 5 \\
\hline $\begin{array}{l}\text { Impacto en el aprendizaje de los estudiantes al usar las herra- } \\
\text { mientas }\end{array}$ & 9 & 4 & 0,894 & 0,917 & 1 & 5 \\
\hline Expectativas del estudiante & $10 \_\mathrm{a}$ & 4 & 0,290 & 0,539 & 3 & 5 \\
\hline Actitud del estudiante & $10 \_b$ & 4 & 0,360 & 0,600 & 3 & 5 \\
\hline $\begin{array}{l}\text { Contribución al desarrollo del pensamiento crítico en los } \\
\text { estudiantes }\end{array}$ & $10 \_c$ & 4 & 0,490 & 0,700 & 2 & 5 \\
\hline Comunicación del estudiante & $10 \_d$ & 4 & 0,240 & 0,490 & 4 & 5 \\
\hline $\begin{array}{l}\text { Creatividad e innovación en la solución de problemas por parte } \\
\text { del estudiante }\end{array}$ & 10_e & 5 & 0,450 & 0,671 & 3 & 5 \\
\hline Interés del estudiante & $10 \_f$ & 4 & 0,800 & 0,894 & 3 & 5 \\
\hline Motivación del estudiante & $10 \_\mathrm{g}$ & 4 & 0,490 & 0,700 & 3 & 5 \\
\hline Actitud del docente & $11 \_\mathrm{a}$ & 5 & 0,440 & 0,663 & 3 & 5 \\
\hline Interés del docente & 11_b & 5 & 0,410 & 0,640 & 3 & 5 \\
\hline Motivación del docente & $11 \_c$ & 5 & 0,240 & 0,490 & 4 & 5 \\
\hline
\end{tabular}

Fuente: elaboración propia. 


\section{Caracterización de los docentes}

La primera parte del instrumento correspondió a preguntas de caracterización, entre estas las que trataban sobre el género del docente: hubo cinco hombres y cinco mujeres. De manera reflexiva se encuentra una proporción igualitaria en cuanto a la participación por género.

Respecto de la edad de los docentes, se encontró que los tres docentes que están en el rango de edad de 21 años a 30 años son mujeres. De cinco docentes en el rango de edad de 31 años a 40 años, una es mujer y cuatro, hombres. Hubo un docente en el rango de edad de 41 años a 50 años y una docente en el rango de edad de 51 años a 60 años. No hubo docentes menores de 20 años ni mayores de 60 años.

En relación con la distribución de la modalidad de enseñanza seleccionada por los docentes, se encontró que ellos eligieron la modalidad en la cual poseían una mayor carga académica. A partir de las respuestas, se identificó que cinco docentes enseñaban en la modalidad semipresencial, de los cuales cuatro eran mujeres y uno, hombre; tres docentes instruían en modalidad virtual, esto es, dos hombres y una mujer, y dos docentes enseñaban en modalidad presencial, en la que las TIC son usadas para apoyar a la enseñanza, los cuales son dos hombres de edades entre 31 años y 40 años. Que los docentes hayan seleccionado la modalidad de enseñanza con mayor carga horaria no los excluye de ser docentes que también tienen cursos en modalidad virtual.

\section{Calidad de la capacitación}

La segunda parte de la encuesta correspondió a la valoración de la capacitación, para lo cual se les preguntó a los docentes si esta cumplió con sus expectativas: nueve docentes respondieron afirmativamente y uno, negativamente. El docente que consideró que no se cumplió con sus expectativas expresó que esperaba que el taller le "brindara contenido extra clase para fortalecer la adquisición de competencias por parte del alumno" y su curso fue orientado en la modalidad presencial.

La figura 2 contiene la síntesis de las respuestas obtenidas por los docentes frente a las expectativas que tenían de la capacitación. En general, las expectativas eran acerca del aprendizaje, el conocimiento, el uso y la actualización en herramientas informáticas para enriquecer la práctica docente, además de esperar que las herramientas sirvieran como apoyo a la labor docente para facilitar la enseñanza.

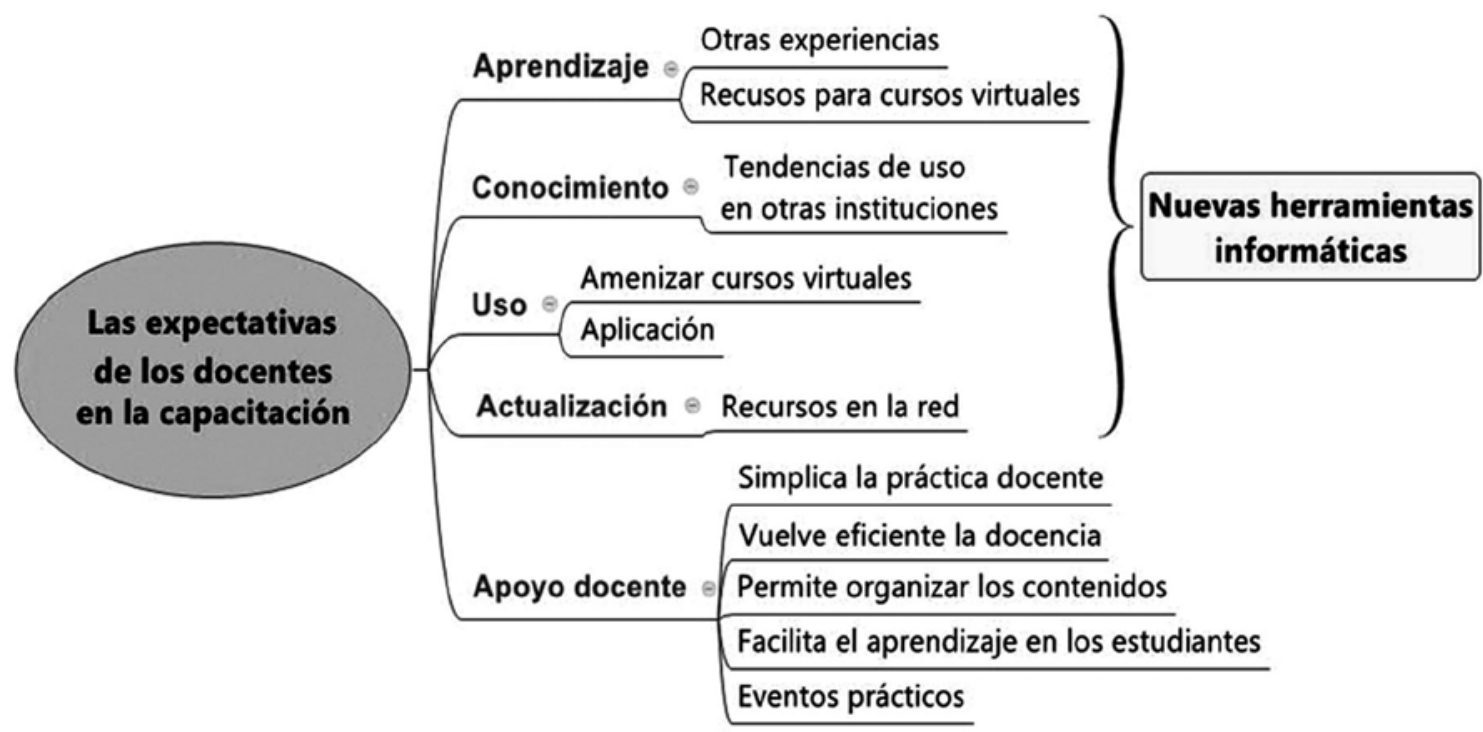

Figura 2. Síntesis de respuestas. Expectativas de la capacitación.

Fuente: elaboración propia. 
Se indagó sobre la motivación de buscar otras herramientas de software para apoyar el quehacer educativo que le dejó la capacitación al docente. Se encontró que seis docentes respondieron que sí estaban motivados y cuatro docentes respondieron que no. Los docentes que respondieron positivamente se distribuyen en cinco hombres y una mujer. Esta última imparte su curso en la modalidad semipresencial, y los hombres orientaron cursos así: dos en modalidad virtual, dos en modalidad presencial y uno en modalidad semipresencial. Por otro lado, los docentes que respondieron negativamente son mujeres: tres de ellas orientaron un curso en modalidad semipresencial y una en modalidad virtual. Las herramientas adicionales que encontraron los docentes para el desarrollo de sus clases se listan a continuación:

\begin{tabular}{|c|c|c|}
\hline - Aplicaciones en & -Geometry Pad ${ }^{\circledR}$. & -Quicktime ${ }^{\oplus}$. \\
\hline $\mathrm{Java}^{\oplus}$ & • Gliffy ${ }^{\oplus}$ & •Quick- \\
\hline - BlogSpot ${ }^{\oplus}$. & - Glogster ${ }^{\circledast}$. & graph $^{\oplus}$. \\
\hline • Diigo ${ }^{\circledast}$. & -Google Drive ${ }^{\circledast}$ & -Toondoo ${ }^{\circledR}$. \\
\hline - Dropbox ${ }^{\oplus}$. & - Google Forms. & •Tumblr \\
\hline - Edmodo ${ }^{\oplus}$. & - Movenote ${ }^{\circledR}$. & -Wolfram/ \\
\hline •Evernote ${ }^{\circledast}$ & •Prezi & Alpha ${ }^{\oplus}$ \\
\hline
\end{tabular}

Posteriormente, se analizó la utilidad de la capacitación para su aplicación en cursos en línea. Las respuestas obtenidas en escala tipo Likert muestran que no hubo datos para los niveles 1, "inútil", y 2, "poco útil”. Para el nivel 3, "moderadamente útil”, se registró una respuesta; para el nivel 4, "útil", se registraron tres respuestas, y para el nivel 5, "muy útil”, se registraron seis respuestas. Estas respuestas permiten apreciar que las capacitaciones en herramientas informáticas son siempre bienvenidas, toda vez que contribuyen a mejorar el quehacer educativo.

\section{Herramientas}

La tercera parte de la encuesta permitió determinar la facilidad y el uso de las herramientas por parte de los docentes. Esta variable se compuso de dos preguntas que especifican cada uno de los programas informáticos usados en la capacitación. Se les preguntó a los docentes por la herramienta informática que más les gustó teniendo en cuenta la facilidad de uso. Para la medición se empleó la siguiente escala: 1. muy difícil, 2. difícil, 3. moderadamente fácil, 4. fácil y 5. muy fácil (ver tabla 4).

En esta sección, las respuestas fueron en su mayoría positivas, fueron de "moderadamente fácil" a "muy fácil”, que es lo mismo que del nivel 3 al 5 . La herramienta que los participantes percibieron más sencilla fue la de Letreros: ocho participantes la ubicaron en el nivel 5. Por otro lado, se consideraron las herramientas de evaluación y contenidos escolares o integradores como las más complejas: ocho docentes la situaron en el nivel 3 y dos, en el nivel 1, considerándola "muy difícil".

Tabla 4. Respuestas a la pregunta 7: ¿cuál de las herramientas presentadas en la capacitación le gustó más teniendo en cuenta su facilidad de uso?

\begin{tabular}{|l|c|c|c|c|c|c|}
\hline \multicolumn{1}{|c|}{ Ítem } & $\mathbf{1}$ & $\mathbf{2}$ & $\mathbf{3}$ & $\mathbf{4}$ & $\mathbf{5}$ & $\mathbf{N}$ \\
\hline Letreros & 0 & 0 & 0 & 2 & 8 & 10 \\
\hline Esquemas & 0 & 0 & 2 & 5 & 3 & 10 \\
\hline Cómics & 0 & 0 & 2 & 6 & 2 & 10 \\
\hline Videos y flash & 0 & 0 & 0 & 5 & 4 & 9 \\
\hline Evaluación & 1 & 0 & 3 & 3 & 3 & 10 \\
\hline Contenidos escolares & 0 & 0 & 4 & 2 & 4 & 10 \\
\hline Contenido integrado & 1 & 0 & 1 & 6 & 2 & 10 \\
\hline
\end{tabular}

Fuente: elaboración propia.

Se les preguntó a los docentes por la herramienta informática que más les gustó teniendo en cuenta la utilidad para el curso que orientaban. La escala que se empleó fue la siguiente: 1 . inútil, 2 . poco útil, 3 . moderadamente útil, 4 . útil y 5 . muy útil (ver tabla 5 ).

En la tabla 5 se resaltan las herramientas que en opinión de los docentes tienen una mayor utilidad en sus cursos, en las que los videos y animaciones flash son las de mayor valoración, toda vez que los estudiantes en su mayoría manejan un estilo de aprendizaje visual, y las modalidades de enseñanza son virtuales, por lo que, el desarrollo de video apoya en gran medida una conexión automática y gráfica para la comprensión de los temas. Por otro lado, los esquemas, letreros y cómics presentan un puntaje alto dentro de las preferencias de los docentes, pues tienen las ventajas del aprendizaje lúdico en el que se sigue aprendiendo al motivar la interacción con actividades asociadas al juego o con representaciones gráficas, como un mapa mental o conceptual. Por último, se destaca que un porcentaje bajo de docentes seleccionó los contenidos escolares, integradores y evaluación. Cabe destacar que los resultados pueden estar ligados a que se consideraron herramientas complejas de uso muy difícil (tabla 4). Por lo tanto, de momento no se identifican las grandes ventajas que proporcionan.

Los docentes valoraron su comportamiento y satisfacción al usar alguna de las herramientas de la capacitación como apoyo pedagógico. La escala que se empleó fue la siguiente: 1 . nada satisfecho, 2. poco 
satisfecho, 3. satisfecho, 4. bastante satisfecho y 5 . muy satisfecho (ver tabla 6).

Tabla 5. Respuestas a la pregunta 8: ¿cuál de las herramientas presentadas en la capacitación le gustó más teniendo en cuenta la utilidad en el curso que orienta?

\begin{tabular}{|l|l|l|l|l|l|l|}
\hline \multicolumn{1}{|c|}{ Ítem } & $\mathbf{1}$ & $\mathbf{2}$ & $\mathbf{3}$ & $\mathbf{4}$ & $\mathbf{5}$ & $\mathbf{N}$ \\
\hline Letreros & 1 & 0 & 2 & 3 & 4 & 10 \\
\hline Esquemas & 0 & 0 & 1 & 4 & 5 & 10 \\
\hline Cómics & 0 & 0 & 4 & 2 & 4 & 10 \\
\hline Videos y flash & 0 & 0 & 0 & 3 & 7 & 10 \\
\hline Evaluación & 0 & 2 & 3 & 3 & 2 & 10 \\
\hline Contenidos escolares & 0 & 2 & 2 & 4 & 2 & 10 \\
\hline Contenido integrado & 0 & 1 & 2 & 5 & 2 & 10 \\
\hline
\end{tabular}

Fuente: elaboración propia.

En esta sección se realizó una medición introspectiva de los docentes al seguir utilizando los programas informáticos que aprendieron, en la cual ellos indagaron sobre su propia actuación dentro de la capacitación y después de esta. El “interés” resultó ser la característica que mayor valoración positiva tuvo, lo cual indica que los docentes se encontraban descubriendo nuevas temáticas que lograban capturar su atención. La segunda característica fue la "actitud", y la tercera, la "motivación". Se destaca el entusiasmo provisto a los docentes para usar innovaciones en sus prácticas pedagógicas.

Tabla 6. Respuestas a la pregunta 11: valore su comportamiento (docente) cuando usó alguna de las herramientas de la capacitación como apoyo pedagógico y recursos educativos digitales

\begin{tabular}{|l|l|l|l|l|l|l|}
\hline \multicolumn{1}{|c|}{ Ítem } & $\mathbf{1}$ & $\mathbf{2}$ & $\mathbf{3}$ & $\mathbf{4}$ & $\mathbf{5}$ & $\mathbf{N}$ \\
\hline Actitud & 0 & 0 & 1 & 2 & 7 & 10 \\
\hline Interés & 0 & 0 & 1 & 1 & 8 & 10 \\
\hline Motivación & 0 & 0 & 0 & 4 & 6 & 10 \\
\hline
\end{tabular}

Fuente: elaboración propia.

\section{Efectos de la capacitación}

La cuarta parte del instrumento buscaba conocer según los docentes cuáles habían sido los efectos de la capacitación, al valorar el comportamiento de los estudiantes frente al uso de las Tic. Se le preguntó a los docentes por la herramienta informática que más les gustó teniendo en cuenta el impacto en el aprendizaje de los estudiantes. La escala que se empleó fue la siguiente: 1. bajo impacto: no hubo cambios en el comportamiento de los estudiantes y no fue una experiencia agradable para ellos, y 5 . alto impacto: a los estudiantes les agradó las herramientas y aprendieron con estas. En la tabla 7 se muestra que los letreros son recursos que solo embellecen la presentación de la información en el aula virtual, pero cuatro de los docentes consideran que no tienen un impacto directo en el aprendizaje de los estudiantes, mientras que seis docentes afirman que tienen un impacto alto o medio alto. Nueve docentes consideran de mayor impacto para el aprendizaje de los estudiantes las herramientas informáticas que sirven para la elaboración de esquemas y de videos.

Cinco docentes consideraron de un impacto medio (ni alto ni bajo) las herramientas informáticas para elaborar evaluaciones, es decir, aquellas con una dificultad media; cuatro docentes lo hicieron con un impacto positivo (alto y medio alto), y un docente indica que tiene un impacto medio bajo.

Tabla 7. Respuestas a la pregunta 9: ¿cuál de las herramientas presentadas en la capacitación le gustó más teniendo en cuenta el impacto en el aprendizaje de los estudiantes?

\begin{tabular}{|l|c|c|c|c|c|c|}
\hline \multicolumn{1}{|c|}{ Ítem } & $\mathbf{1}$ & $\mathbf{2}$ & $\mathbf{3}$ & $\mathbf{4}$ & $\mathbf{5}$ & $\mathrm{N}$ \\
\hline Letreros & 1 & 1 & 2 & 3 & 3 & 10 \\
\hline Esquemas & 0 & 0 & 1 & 3 & 6 & 10 \\
\hline Cómics & 0 & 2 & 1 & 5 & 2 & 10 \\
\hline Videos y flash & 0 & 0 & 1 & 3 & 5 & 9 \\
\hline Evaluación & 0 & 1 & 5 & 3 & 1 & 10 \\
\hline Contenidos escolares & 1 & 1 & 3 & 3 & 2 & 10 \\
\hline Contenido integrado & 0 & 1 & 2 & 6 & 1 & 10 \\
\hline
\end{tabular}

Fuente: elaboración propia.

Además, se solicitó a los docentes que valoraran el comportamiento de los estudiantes en el curso cuando usó como apoyo pedagógico alguna de las herramientas sugeridas en la capacitación. La escala que se empleó fue la que sigue: 1 . nada satisfecho, 2 . poco satisfecho, 3. satisfecho, 4 . bastante satisfecho y 5 . muy satisfecho (ver tabla 8 ).

La tabla 8 contiene las reacciones de los estudiantes, valoradas por los docentes dentro de su interacción con los recursos digitales. Dentro de los datos más destacados está que la "creatividad e innovación en la solución" y el "pensamiento crítico" fueron los indicadores con mayor valoración satisfactoria, lo cual es un logro porque los estudiantes son de ingeniería y de diseño. Por lo tanto, el desarrollo de una 
lógica para resolver problemas y las habilidades en torno a la creatividad e innovación son competencias prioritarias en la formación de los docentes. El indicador de "comunicación" también arrojó valoraciones positivas de los docentes, como "bastante satisfecho" y "muy satisfecho". Los indicadores "interés" y "actitud" son valorados por los docentes como de satisfactorio comportamiento por los estudiantes.

Tabla 8. Respuestas a la pregunta 10: valore el comportamiento de los estudiantes en el curso cuando usó alguna de las herramientas de la capacitación como apoyo pedagógico y recursos educativos digitales.

\begin{tabular}{|l|c|c|c|c|c|c|}
\hline \multicolumn{1}{|c|}{ Ítem } & $\mathbf{1}$ & $\mathbf{2}$ & $\mathbf{3}$ & $\mathbf{4}$ & $\mathbf{5}$ & $\mathrm{N}$ \\
\hline Expectativas & 0 & 0 & 2 & 7 & 1 & 10 \\
\hline Actitud & 0 & 0 & 3 & 6 & 1 & 10 \\
\hline Pensamiento crítico & 0 & 1 & 0 & 8 & 1 & 10 \\
\hline Comunicación & 0 & 0 & 0 & 6 & 4 & 10 \\
\hline $\begin{array}{l}\text { Creatividad e innovación en } \\
\text { la solución }\end{array}$ & 0 & 0 & 1 & 3 & 6 & 10 \\
\hline Interés & 0 & 0 & 4 & 2 & 4 & 10 \\
\hline Motivación & 0 & 0 & 2 & 5 & 3 & 10 \\
\hline
\end{tabular}

Fuente: elaboración propia.
Por último, se dejó una pregunta abierta que recogiera las observaciones de los participantes respecto del cuestionario o del proceso de capacitación que recibieron. En general, los comentarios sobre la capacitación fueron positivos, y algunos docentes explicaron los motivos por los cuales no implementaron las herramientas en sus cursos. En la figura 3, se presenta la síntesis de las respuestas obtenidas en la pregunta por las observaciones, que además muestra cuatro categorías para las respuestas asociadas con la instructora de la capacitación, el taller y su contenido, la incorporación de recursos en los cursos virtuales y otros comentarios de agradecimientos y de motivación por continuar el proceso de actualización docente.

Los comentarios de los docentes a los ítems abiertos permiten identificar aspectos que no habían sido recogidos en el cuestionario elaborado, como el impacto del taller y la incorporación de los recursos aprendidos en los cursos que cada docente imparte. Se encontró que, aunque la capacitación les pareció positiva, no incorporaron las herramientas informáticas, dado que los cursos orientados son muy específicos y requieren otro tipo de software.

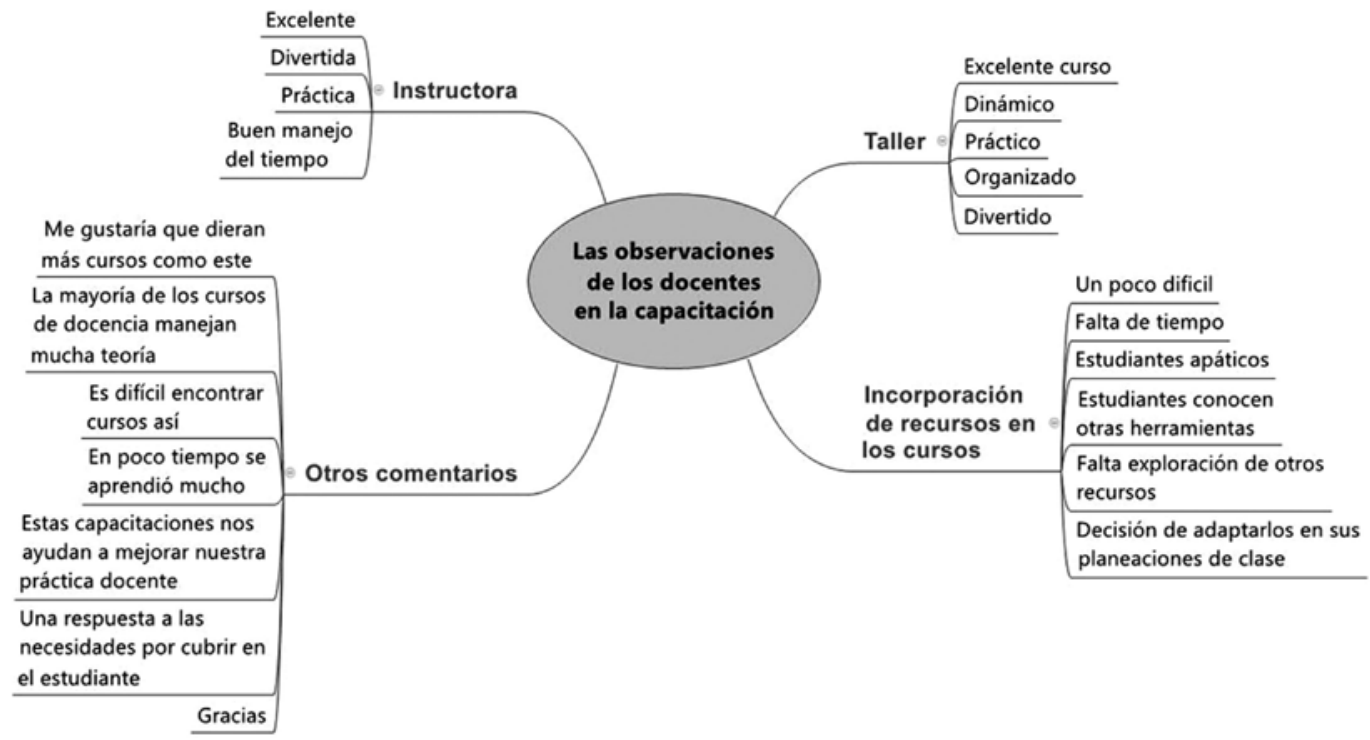

Figura 3. Síntesis de los comentarios generales de los docentes.

Fuente: elaboración propia.

La adquisición de destrezas en el manejo de herramientas y recursos tecnológicos debería incorporarse en las unidades de aprendizaje como estrategia de actualización de los planes y programas de estudio. Así se puede atender a nuevos paradigmas pedagógicos en los que se promueve el uso de las TIC en prácticas innovadoras del aprendizaje o en procesos administrativos propios de la labor docente, como 
la utilización de redes y espacios colaborativos entre colegas, y el desarrollo de tutorías o asesorías con estudiantes (extraclase). Se debe ser conscientes de que el uso de las TIC no solo se debe promover para el desarrollo de estrategias innovadoras de enseñanzaaprendizaje, sino también en la gestión de datos e información y el desarrollo profesional de docente.

\section{Discusión y conclusiones}

A partir de la experiencia se encontró que los docentes valoran positivamente la actualización permanente a través de talleres prácticos para la incorporación de TIC en sus actividades de enseñanza. Las capacitaciones en TIC permiten que los profesores encuentren en estas un complemento para su acción docente (Álvarez et al. 10). En Latinoamérica, a diferencia de Europa, la inclusión de las Tic en las prácticas educativas no es una imposición externa, sino que es una iniciativa propia de cada institución de educación superior.

Proveer en las capacitaciones otras herramientas informáticas más específicas (o complejas) en el apoyo a la práctica de enseñanza genera una mejor actitud y un mayor interés por parte de los docentes, toda vez que verán en los programas informáticos mayor utilidad y aplicación directa en sus aulas virtuales sin tener que realizar desarrollos, solo usar las herramientas tal como las presenta Internet. Es claro que el cambio de actitud e interés de los docentes hacia el uso de las TIC en sus prácticas educativas depende de la integración que puedan hacer. Un hallazgo similar encontraron Caicedo-Tamayo y Rojas-Ospina (528), quienes especifican que cada vez más los docentes están abiertos a aprender nuevas herramientas o a apropiar y aprovechar mejor las ya conocidas.

De acuerdo con Álvarez et al., se puede decir que las actitudes de los docentes, la motivación, el interés, las expectativas, la experiencia sociocultural o la ansiedad, se engloban dentro de las denominadas "variables afectivas de aprendizaje", que afectan su aprendizaje cuando participan de actividades de formación y pueden influir de manera indirecta en el aprendizaje de los estudiantes que tienen a su cargo (3).

Todos los participantes consideran que las TIC pueden ayudar a mejorar sus actividades de enseñanza, organizar los contenidos, organizar los recursos educativos de los cursos y pueden tener los beneficios per se que posee el uso de la tecnología. Sobre la actitud positiva hacia las TIC coinciden los estudios de
Álvarez et al. (11), Caicedo-Tamayo y Rojas-Ospina (528) y Riascos-Erazo, Quintero-Calvache y ÁvilaFajardo (134-146).

Lo que se obtuvo de la evaluación de la formación docente mostró resultados favorables respecto a la incorporación y uso de recursos didácticos por parte de los docentes. También se dio una mayor motivación e interés por realizar capacitaciones en TIC con mayor complejidad o más específicas, y se generó un estímulo hacia el autoaprendizaje que se verifica en la búsqueda y el conocimiento de nuevos recursos digitales para apoyar su práctica docente. En la opinión de los docentes sobre al aprendizaje de los estudiantes, ellos se muestran abiertos al uso de herramientas novedosas y visualizan una aplicación real en su proceso de aprendizaje. El desarrollo de competencias y habilidades de interés en las áreas de ingeniería y diseño, como la creatividad, el pensamiento crítico, la innovación y la comunicación, se potencializan al incorporar recursos didácticos digitales en las estrategias de enseñanza.

Somos conscientes de que la cantidad de docentes participantes de la capacitación y la de la formación es baja; por ende, esta no es representativa. Sin embargo, para los fines de esta investigación, los de contar la experiencia y realizar su evaluación un año después de desarrollada, es meritorio: nos permitió conocer el efecto que la capacitación tuvo en los docentes y si estos aplicaron o no las herramientas aprendidas, pero lo más destacable es que motivó por lo menos a la mitad de los docentes a realizar búsquedas sobre nuevos programas informáticos que sirvieran a sus fines puntuales de enseñanza. Consideramos relevante destacar que en comparación con el trabajo de Álvarez et al. nuestro trabajo tuvo en cuenta la caracterización docente (género, edad, modalidad de enseñanza), y los resultados obtenidos son coherentes con los presentados en ese estudio.

Encontramos loable que en el diseño de eventos académicos para docentes se logren las expectativas y se atiendan las necesidades reales. De esta manera el docente mantiene el interés por una formación constante y la institución educativa contribuye con el mejoramiento de la calidad de sus programas y sus estudiantes. Así, las iniciativas institucionales por cerrar la brecha entre lo proyectado en los planes de desarrollo institucional y los objetivos de cada docente en la integración de las TIC y sus usos reales es un hecho.

Por último, se resalta la importancia en el desarrollo de la oferta de capacitación a docentes como 
una estrategia para socializar temas de tecnología que impacten directamente en sus programas académicos. Así mismo se consideran valiosas la interacción y la cooperación entre docentes al compartir experiencias y acuerdos para la impartición de catedra lo cual impacta en la motivación del docente.

\section{Agradecimientos}

Norma Candolfi Arballo agradece a la Universidad Autónoma de Baja California por el apoyo en la convocatoria de movilidad académica del 2013 que permitió la estancia de la Mtra. Diana Marcela Cardona de la Universidad Nacional de Colombia, quien fungió como instructora del Taller Básico para crear Recursos Educativos Digitales.

Diana Marcela Cardona Román agradece a Colciencias por el apoyo a su formación doctoral a través de la convocatoria de doctorados nacionales n. ${ }^{\circ} 567$ del 2012.

\section{Referencias}

Alonso Tejada, María Erendira. "La planeación didáctica". Cuadernos de Formación de Profesores 3 (2009): 1-10.

Álvarez, Susana et al. "Actitudes de los profesores ante la integración de las TIC en la práctica docente: estudio de un grupo de la Universidad de Valladolid". Edutec. Revista Electrónica de Tecnología Educativa 35 (2011): 1-19. Web.

Caicedo-Tamayo, Adriana María y Tatiana Rojas-Ospina. "Creencias, conocimientos y usos de las TIC de los profesores universitarios". Revista Educación y Educadores 17.3 (2014): 517-533. Impreso. doi: 10.5294/ edu.2014.17.3.7.

Cardona-Román, Diana Marcela y Jenny Marcela Sánchez-Torres. "Descripción de los Indicadores e-learning en el Marco de los Sistemas de Evaluación de la Sociedad de la Información". Tenth LACCEI Latin American and Caribbean Conference for Engineering and Technology (LACCEI 2012): Megaprojects: Building Infrastructure by Fostering Engineering Collaboration, Efficient and Effective Integration and Innovative Planning. Ed. Universidad de Panamá. Panamá: LACCEI, 2012. Web.

CEA. Programa flexible de formación y desarrollo docente. Documento Institucional. Baja California. México: Centro de Educación Abierta de la Universidad Autónoma de Baja California, 2015. Web. <http://cea.mxl. uabc.mx/capacitacion/programa-de-capacitacion-pffdd $>$.

Creswell, John W. Research Design qualitative, quantitative, and mixed methods approaches. Tercera edición. Los Angeles: Sage, 2008. Impreso.

Dávila, Gianina et al. "Conocimiento práctico de los profesores: sus características y contradicciones en el contexto universitario actual". Revista de la Educación Superior 42.2 (2013): 35-53. Web.

Gisbert-Cervera, Mercè. "El profesor del siglo xxi: de transmisor de contenidos a guía del ciberespacio". Web. 20 de agosto del 2015. < http://tecnologiaedu.us.es/cuestionario/bibliovir/203.pdf $>$

González, Julio Cesar. “Tic y la transformación de la práctica educativa en el contexto de las sociedades del conocimiento". Revista de Universidad y Sociedad de Conocimiento 5.2 (2008): 1-8. Impreso.

González-Such, José, Margarita Bakieva y Rosario García-Bellido. "Análisis de un cuestionario para evaluar materiales elaborados por personal docente en un espacio colaborativo para la innovación educativa". Revista Iberoamericana de Evaluación Educativa 7.2 (2014): 223-237. Web.

IDIE. Paradojas en la formación docente: Elementos para avanzar en su reflexión y planteamiento de propuestas. Ed. Mireya González-Lara. Vol. 1. Bogotá, Colombia: IDIE; OEI; Grupo sM; Gobierno de Aragón, 2008. Impreso.

Monroy-Farías, Miguel. "La planeación didáctica”. Ed. Miguel Monroy-Farías, Ofelia Contreras-Gutiérrez y Ofelia Desatnik-Miechimsky. Psicología educativa. Iztacala, México: UNAM; Facultad de Estudios Superiores Iztacala, 2009. 453-487. Impreso.

Morone, Guillermo. Métodos y técnicas de la investigación científica. Documento de trabajo. Valparaíso, Chile: Pontificia Universidad Católica de Valparaíso, 2013. Impreso.

OEI. Los desafíos de las TIC para el cambio educativo. Ed. Roberto Carneiro, Juan Carlos Toscano y Tamara Díaz. Madrid, España: Organización de Estados Iberoamericanos para la Educación la Ciencia y la Cultura, 2009. Impreso.

Paniagua, María Eugenia. La formación y la actualización de los docentes: herramientas para el cambio en educación. San José: Centro de Estudios Democráticos de América Latina, 2004. Web.

Riascos-Erazo, Sandra Cristina, Diana María Quintero-Calvache y Gloria Patricia Ávila-Fajardo. "Las TIC en el 
aula: percepciones de los profesores universitarios". Educación y Educadores 12.3 (2009): 133-157. Impreso

Severin, Eugenio. Tecnologías de la información y la comunicación (TIC) en Educación. Marco Conceptual e Indicadores. Vol. 6. Washington: Banco Interamericano de Desarrollo, 2010. Impreso.

UABC. Plan de Desarrollo Institucional PDI 2011-2015. Institucional. Baja California, México: Universidad Autónoma de Baja California, 2011. Web. 20 de agosto de 2015. <http://www.uabc.mx/planeacion/pdi/20112015/pdi2011.pdf $>$.

Unesco. "Aumento de las competencias de los docentes en materia de TIC gracias a los recursos educativos abiertos". Communication \& Information Sector. 3 de julio del 2013: 1. Impreso.
-. Estándares de competencia en TIC para docentes. Londres, Inglaterra: Unesco, 2008. Web. 20 de agosto del 2015. $<$ http://www.oei.es/tic/UNESCOEstandaresDocentes. pdf $>$.

Unesco y IEu. Medición de las tecnologías de la información y la comunicación (TIC) en educación: Manual del usuario. Documento Técnico n. ${ }^{\circ}$ 2. Montreal, Canadá: Unesco Institute for Statistics, 2010.

Vezub, Lea F. "La formación y el desarrollo profesional docente frente a los nuevos desafíos de la escolaridad. Profesorado. Revista de Currículum y Formación del Profesorado 11.1 (2007): 23. Impreso.

Wake, Jo, Olga Dysthe y Stig Mjelstad. "New and Changing Teacher Roles in Higher Education in a Digital Age". Educational Technology \& Society (2007): 40-51. 